\title{
SUPERCONDUCTIVITY AND MAGNETISM AT NUCLEAR-MATTER DENSITIES: AN ASTRONOMICAL CHALLENGE
}

\author{
M. JAHAN-MIRI \\ Institute for Advanced Studies in Basic Sciences \\ Zanjan 45195, Iran \\ E-mail: jahan@iasbs.ac.ir
}

\begin{abstract}
We report on a study of the evolution of magnetic fields of neutron stars, driven by the expulsion of magnetic flux out of the proton superconducting core of the star. The rate of expulsion, or equivalently the velocity of outward motion of fluxcarrying proton-vortices is determined from a solution of their equation of motion. A determination of the effective forces on the fluxoids moving through the quantum liquid interior of neutron stars is however confronted with many ambiguities about the properties of this special case of superconductivity in the nature. Also, the behaviour of the fluxoids at the core boundary, and the subsequent evolution of the expelled flux within the highly conductive surrounding crust, are other related issues that have not been so far explored in any great details.
\end{abstract}

\section{Introduction}

Observational evidence has accumulated that magnetic fields of neutron stars decay over time scales of tens of million years or more 27,20. Theoretical modelling of such a field decay calls for a study of the expulsion of magnetic flux out of the superconducting core of the star, which is embedded within the highly conductive crust of the star. The study would bear on an understanding of the magnetic and superconductivity properties of matter under conditions much different than is usually realised for the terrestrial superconductors. Some of the novelties might be listed:

- type I/II proton superconductivity; normal electrons,

- "field cooling" in an intrinsic field, originally supported by electron currents,

- coherent scattering of electrons off fluxoids,

- "transfer" of flux from proton super-currents to electron currents outside,

- freezing of fluxoids end points; geometry of the expelled flux, and

- spherical geometry and $\mathrm{km}$ size of the superconductor and fluxoid lattice.

The velocity of outward motion of flux-carrying proton-vortices (fluxoids) may be determined from a solution of the Magnus equation of motion for these vortices 19.22 , taking into account the various forces that act on them. For the terrestrial type-II supercunductors flux movement is usually driven by the Lorentz force due to an applied transport current. In contrast, in the interior of a neutron star there are other forces acting on the fluxoids. These include $i$ ) a force due to their pinning interaction with the moving neutron vortices, ii) viscous drag force due to magnetic scattering of electrons, iii) buoyancy force, and iv) curvature force. The derived radial velocity of the fluxoids at the core-crust boundary would in turn determine the rate of the flux expulsion out of the core. The magnetic evolution of the star may be thus determined by further following the Ohmic dissipation of the expelled flux within the crust. A detailed description of the modelling of such a flux expulsion and field evolution and the astrophysical implications of the results of our computations is reported elsewhere 13, 2 . 2 . 2 . Here we give a brief outline of aspects of the models directly relevant to the superconductivity effects. Our aim would be to also 
highlight the basic issues, listed above, which require further theoretical investigations pertaining to the superconductivity and magnetism in neutron stars.

\section{The Superconductor}

All theoretical models of neutron stars predict a (quantum) liquid interior ("core") at densities $\left(\sim 2.4 \times 10^{14} \mathrm{~g} \mathrm{~cm}^{-3}\right)$ similar and above that of the nuclear matter, consisting of bare neutrons plus an admixture of protons and electrons, which is surrounded by a solid metallic crust of neutron-rich nuclei and relativistic degenerate electrons 1.23.21. Neutron stars are "cold" objects, having internal temperatures $T \sim 10^{8}(\mathrm{~K})<<T_{\text {Fermi }} \sim 10^{12} \mathrm{~K}$. For the same theoretical reasons that terrestrial matter is argued to become superconducting with a transition temperature $T$ $10^{-3} T_{\text {Fermi }}$ the neutron stars are predicted to have superfluid-superconductor interiors 6.9.28. Cooper pairs, in a neutron star, are however formed due to the long-range attractive part of nucleon-nucleon interaction among protons, as well as neutrons. The proton component of the core is usually assumed to form a type-II superconductor, based on its estimated values of the penetration depth $\lambda_{\mathrm{p}} \sim 10^{2} \mathrm{fm}$ and the coherence length $\xi_{\mathrm{p}} \sim$ few fm. The possibility of type-I proton superconductivity, $\xi_{\mathrm{p}}>\sqrt{2} \lambda_{\mathrm{p}}$, has been also considered, allowing for the existing uncertainities in the values of density, effective mass of protons, and transition temperature in the core 1.7 . However, no attempts have been reported on the modelling of the field evolution of the star for the case of type-I superconductivity. The transition to the superconducting state is believed to occur at a constant field, originally present in the core in its normal statet. This should lead to formation of a lattice of proton flux tubes (fluxoids) which extend some few $\mathrm{km}$ across the spherical core of the star. Distorted geometries of lines might be also realized due to the presence of field components other than a pure dipolar field in the normal state $\mathrm{E}$, which is neglected in our models for simplicity. An interesting feature of this flux trapping is that, in contrast to the usual requirement for the Meissner effect, the field need not be expelled in spite of being smaller than the associated lower critical value $H_{\mathrm{c} 1} \sim 10^{15} \mathrm{G}$, which is indeed the case for the typical field strengths of neutron stars $\sim 10^{12} \mathrm{G}$. The large conductivity of the matter in the normal state implies dissipation time scales, for the electron currents in the core, many orders of magnitudes larger than the time taken for the star to cool down below its transition temperature $T_{\mathrm{c}}$. This is argyed to imply freezing in of the flux even at field strengths smaller than the lower critical valuet 11 .

In the thin layer $(\sim 1 \mathrm{~km})$ of the crust, surrounding the superconductor core, matter at the density range $7 \times 10^{6}\left(\mathrm{~g} \mathrm{~cm}^{-3}\right) \lesssim \rho \lesssim 2.4 \times 10^{14}\left(\mathrm{~g} \mathrm{~cm}^{-3}\right)$ is highly conducting. The estimated Ohmic dissipation time for the crust, due to electron scattering by phonons and lattice impurities is uncertain and lies in the range $10^{7}-10^{9} \mathrm{yr}$. Moreover, the unknown geometry of the expelled flux and its transport behavior within the crust further complicates the calculation of its decay time scale 15. The distinctive features of the flux expulsion in the present case, in contrast to that of the terrestrial superconductors, should be born in mind. Firstly, this is not an expulsion of the flux associated with an externally applied field that might be understood in terms of interaction of the fluxoid current with the London supercurrent at the surface, and a readjustment of the latter. The flux expulsion out of the core of neutron stars requires electron currents to be induced in the surrounding crust, at the cost of proton supercurrents of the fluxoids being annihilated. Furthermore, the fluxoids end points spout out in a highly conductive surrounding medium. Considering the spherical geometry of the superconductor, it is not clear whether the expulsion of the flux is realised only at the magnetic equator or elsewhere as well. Also, the back reaction of the flux accumulated in the crust, as well as the effect of a surface "barrier", on the fluxoids motion close to the boundary might have significant new results. For example, the surface barrier for the present case, having external fields smaller than $H_{\mathrm{c} 1}$, should be an outward declining hill causing the fluxoids to be drained out of the superconductor 3 . Such an expulsion mechanism due to surface effects has not been so far addressed, in the case of neutron stars; one should see 
how the corresponding rate of flux expulsion, if it is realized, compares with that due to the body forces on fluxoids which are discussed here.

\section{Effective Forces on Fluxoids}

\subsection{Pinning Force}

The rotation of the neutron superfluid implies that there exist angular-momentum-carrying neutron-vortex lines within the core of a neutron star. These are believed to form a regular lattice parallel to the rotation axis of the star which is in general tilted with respect to the magnetic axis, ie. the direction of the fluxoids. Thus, a neutron vortex might presept a "pinning site" against a moving fluxoid, vice versa, should the two structures overlap 28 25. 26; some $10^{31}$ fluxoids and $10^{16}$ vortices should be, typically, present in a neutron star. In the steady-state, the n-vortices must be co-rotating with the charged component of the star, including the lattice of the p-fluxoids, at a rate $\Omega$. For a given rate $\dot{\Omega}$ of spinning down of the star the radial velocity $v_{\mathrm{n}}$ of the n-vortex outward motion, at the core boundary, would be

$$
v_{\mathrm{n}}=-\frac{R_{\mathrm{c}}}{2} \frac{\dot{\Omega}}{\Omega_{\mathrm{s}}} \approx-\frac{R_{\mathrm{c}}}{2} \frac{\dot{\Omega}}{\Omega}
$$

where $R_{\mathrm{c}}\left(\sim 9 \times 10^{5} \mathrm{~cm}\right)$ is the radius of the stellar core, and $\Omega_{\mathrm{s}}(\sim \Omega)$ is the superfluid rotation rate. A superfluid normally spins down while maintaining a positive rotational lag $\omega\left(\equiv \Omega_{\mathrm{s}}-\Omega>\right.$ $0)$ with its vortices. Hence an outward radial Magnus force $F_{\mathrm{M}}=\rho_{\mathrm{s}} \kappa R_{\mathrm{c}} \omega$ would act on the vortices, per unit length at the core boundary, where $\rho_{\mathrm{s}}$ is the neutron-superfluid density, and $\kappa=2 \times 10^{-3} \mathrm{~cm}^{2} \mathrm{~s}^{-1}$ is the vorticity of a vortex line 2 .

The Magnus force on n-vortices has been usually assumed to be balanced by the viscous forces being primarily caused by the scattering of electrons off the magnetized cores of these vortices in the interior of a neutron star 25 . However in presence of the pinning forces it turns out that the latter would be the dominant force which will effectively equate with the Magnus force, resulting in a net zero force on the n-vortices. This force balance condition may be used to calculate the magnitude of the pinning force on n-vortices, as well as its direction which could be, in principle, in either (inward or outward) radial directions depending on the relative motion of the vortices and fluxoids.

The effective pinning force per unit length of each fluxoid may be, in turn, evaluated by noting the equality of the mutual pinning forces between fluxoids and vortices at each pinning site, and then balancing the total pinning force communicated between the two lattices of vortices and fluxoids. However, at any given instant of time only a small fraction of the vortices would be directly interacting with the fluxoids. The remaining much greater fraction of them (of the order of the ratio of an inter-fluxoid spacing to the size of a pinning interaction region; see below) should reside in the inter-fluxoid spacings. Thus the total Magnus force acting continuously on all the vortices may, or may not, be communicated instantaneously to all the fluxoids, depending on the assumed rigidity of the n-vortices and other considerations. We omit a detailed discussion of the derivation of the pinning force on fluxoids for the different possibilities and write down the final results. The pinning force $F_{\mathrm{n}}$ acting on a fluxoid, per unit length, is derived as 12 , either

$$
F_{\mathrm{n}}=\frac{n_{\mathrm{v}}}{n_{\mathrm{f}}} F_{\mathrm{M}} \approx 2 \phi_{0} \rho_{\mathrm{s}} R_{\mathrm{c}} \frac{\Omega(t) \omega(t)}{B_{\mathrm{c}}(t)}=5.03 \frac{\omega_{-6}}{P_{\mathrm{s}} B_{8}} \text { dyncm }^{-1}
$$

where $n_{\mathrm{v}}=\frac{2 \Omega_{\mathrm{s}}}{\kappa}$ and $n_{\mathrm{f}}=\frac{B_{\mathrm{c}}}{\phi_{0}}$ are the number densities per unit cross section area of the vortices and the fluxoids, respectively, $\phi_{0}=2 \times 10^{-7} \mathrm{G} \mathrm{cm}^{2}$ is the magnetic flux carried by a fluxoid, $B_{\mathrm{c}}=10^{8} B_{8}$ is the strength of the core field in units of $\mathrm{G}, \omega_{-6}$ is the superfluid lag $\omega$ in units of $10^{-6} \mathrm{rad} \mathrm{s}^{-1}$, and $P_{\mathrm{s}}$ is the spin period in units of s. Notice that the sign of $\omega$ determines the 
sign of $F_{\mathrm{n}}$ for which, as well as for the other forces discussed below, the outward direction will be reckoned as the positive sense. Or, else,

$$
F_{\mathrm{n}}=\frac{d_{\mathrm{P}}}{d_{\mathrm{f}}}\left(\frac{n_{\mathrm{v}}}{n_{\mathrm{f}}} F_{\mathrm{M}}\right)=2.59 \times 10^{-4} \frac{\omega_{-6}}{P_{\mathrm{s}} B_{8}^{1 / 2}}{\mathrm{dyn} \mathrm{cm}^{-1}}^{-1}
$$

where $d_{\mathrm{f}}=2.3 \times 10^{-7} B_{8}^{-\frac{1}{2}} \mathrm{~cm}$ is the inter-fluxoid spacing, and $d_{\mathrm{P}}$ is the effective size of a pinning interaction region around each fluxoid. A value of $d_{\mathrm{P}}=\lambda_{\mathrm{p}}^{*}=118 \mathrm{fm}$ has been used for the assumed magnetic pinning mechanism (see below), where $\lambda_{\mathrm{p}}^{*}$ is the effective London length of the proton superconductor, being also a length scale for the spread of the magnetic field of a neutron vortex line.

\section{Critical lag:}

The magnitude of the force which could be exerted at each intersection by a vortex on a fluxoid, and vice versa, is limited by a maximum value $f_{\mathrm{P}}$ corresponding to the given strength of the pinning energy $E_{\mathrm{P}}$ and the finite length scale of the interaction $d_{\mathrm{P}}$; namely $E_{\mathrm{P}}=f_{\mathrm{P}} d_{\mathrm{P}}$. This implies a maximum limiting value that the Magnus force on the n-vortices could achieve, corresponding to a maximum critical lag $\omega_{\text {cr }}$ which is given as

$$
\omega_{\mathrm{cr}}=1.59 \times 10^{-6} B_{8}^{1 / 2} \mathrm{rad} \mathrm{s}^{-1}
$$

The critical lag is the magnitude of the lag when radial velocities of fluxoids and vortices are different; ie. $\omega=\omega_{\text {cr }}$ or $\omega=-\omega_{\text {cr }}$ when the vortices move faster or slower than the fluxoids, respectively. However, during a co-moving phase when the force communicated between a vortex and a fluxoid at each pinning point is less than its maximum value, $f_{\mathrm{P}}$, the lag might have any value within the range $-\omega_{\mathrm{cr}}<\omega<\omega_{\mathrm{cr}}$. The pinning energy arising from the magnetic interaction of a fluxoid-vortex pair is estimated to be $E_{\mathrm{P}} \sim 10^{-5}$ ergs. A different estimate for the pinning energy, due to the proton density perturbation, gives a smaller value of $E_{\mathrm{P}} \sim 5 \times 10^{-7}$ ergs 25.16. However, both mechanisms result in similar values for the pinning force, hence similar $\omega_{\mathrm{cr}}$, since the interaction length, $d_{\mathrm{P}}$, for the latter $\left(=\xi_{\mathrm{p}}\right)$ is smaller than for the magnetic interaction $\left(=\lambda_{\mathrm{p}}^{*}\right)$ by about the same ratio as the inverse of the pinning energies.

\subsection{Drag force}

An isolated fluxoid moving through the normal degenerate electron gas in the core of a neutron star is subject to the viscous drag force of the electrons scatterino off its magnetic field. The viscous drag force, per unit length of a fluxoid, is estimated to be睡

$$
\overrightarrow{F_{v}}=-\frac{3 \pi}{64} \frac{n_{\mathrm{e}} e^{2} \phi_{0}^{2}}{E_{\mathrm{F}_{\mathrm{e}}} \lambda_{\mathrm{p}}} \frac{\overrightarrow{v_{\mathrm{p}}}}{c}=-7.30 \times 10^{7} \overrightarrow{v_{\mathrm{p}}} \text { dyn } \mathrm{cm}^{-1}
$$

where $v_{\mathrm{p}}$ is the velocity of the outward radial motion of the fluxoids in units of $\mathrm{cm} \mathrm{s}^{-1}, n_{\mathrm{e}}=$ $3 . \times 10^{36} \mathrm{~cm}^{-3}$ is the number density of the electrons, and $E_{\mathrm{F}_{\mathrm{e}}}=88 \mathrm{MeV}$ is the electron Fermi energy, corresponding to a total density $\rho=2 \times 10^{14} \mathrm{~g} \mathrm{~cm}^{-3}$, and a neutron number density $n_{\mathrm{n}}=1.7 \times 10^{38} \mathrm{~cm}^{-3}$ in the core.

The expression for $F_{v}$ in Eq. 5 is derived based on the assumption of independent motions for single fluxoids. However, for the typical conditions in the interior of a neutron star the lattice of fluxoids might be, as a whole (or at least as bundles consisting of not less than ten million fluxoids), "frozen-in" the electron gasl. This is because the mean distance between the successive magnetic scattering of electrons by fluxoids turns out to be many orders of magnitudes smaller than the mean free path of the electrons due to other events, and also that the deflection angle 
at each scattering event is very small. A detailed treatment of the coherent electron scattering by the fluxoid lattice has been shown to indeed require an almost zero relative velocity between the electrons and the lattice. Therefore, the flux expulsion out of the core might be prohibited altogether except if electron-current loops across the core-crust boundary is realized. Uncertainties about the true distribution of the magnetic flux and the correct value of the conductivity in the crust, and also the possibility of a mechanical failure of the solid crust due to a build-up of the magnetic stresses, however, obscure any definite conclusion to be drawn 14 .

Moreover, there are other reasons to suspect the above suggested frozen-in approximation for the fluxoids as a whole 24 . For example, the finite volume of the fluxoid lattice and also the influence of the superconductor boundary effects on the motion of the fluxoids which have not been included in the above mentioned studies of the coherent scattering could as well have significant new consequences. In addition, the motion of the incompressible electron fluid in the interior of a neutron star has been argued to be divergence freet. Any motion of the fluxoids along with the electrons, in the frozen-in approximation, must be, therefore, of the same (divergencefree) nature. This is however impossible for the uniform lattice of fluxoids during its outward motion since the lattice constant keeps changing. Hence, a compromise between the flux freezing and the divergence-free motion of the electrons has to be worked out if any flux expulsion is to be accounted for.

Given the above uncertainties, as well as the lack of any other definite prescription for calculating the drag force due to the electron scattering, we use a value of $F_{v}$ as given by Eq. 5 in our models. This choice is also supported by noticing that a tentative expulsion time scale derived for the case of coherent scattering, as implied by the Hall drift of the flux at the base of the crust, turns out to be similar to that based on the single fluxoid approximation 15.16 .

\subsection{Buoyancy force}

The buoyancy force on fluxoids in a neutron star arises for reasons analogous to the case of macroscopic flux tubes in ordinary stars. Because flux tubes are in pressure equilibrium with their surrounding the excess magnetic pressure causes a deficit in the thermal pressure, and hence in the density, of the plasma inside a flux tube which make the tube to become buoyant The radially outward buoyancy force $F_{\mathrm{b}}$ on a fluxoid, per unit length, can be expressed as 18.14

$$
F_{\mathrm{b}}=\left(\frac{\phi_{0}}{4 \pi \lambda_{\mathrm{p}}}\right)^{2} \frac{\ln \left(\lambda_{\mathrm{p}} / \xi_{\mathrm{p}}\right)}{R_{\mathrm{c}}}=0.51 \mathrm{dyn} \mathrm{cm}^{-1}
$$

\subsection{Curvature force}

The tension of a vortex line (such as a fluxoid) implies that a curved geometry of the line would result in a restoring force, the curvature force, which tries to bring the line back to its minimum energy straight configuration. The concavely directed curvature force $F_{\mathrm{c}}$, per unit length, on a vortex having a tension $T$ and a curvature radius $S$ is given as $F_{\mathrm{c}}=T / S$. An outward moving fluxoid might be bent outward and becomes subject to an inward curvature force, since its end points are frozen in at the highly conductive bottom of the crust. Thus, for a fluxoid with a tension $T_{\mathrm{p}}=\left(\phi_{0} / 4 \pi \lambda_{\mathrm{p}}\right)^{2} \ln \left(\lambda_{\mathrm{p}} / \xi_{\mathrm{p}}\right)$ the curvature force, per unit length, would be given as $\mathrm{G}$.

$$
F_{\mathrm{c}}=-\frac{R_{\mathrm{c}}}{S}\left(\frac{\phi_{0}}{4 \pi \lambda_{\mathrm{p}}}\right)^{2} \frac{\ln \left(\lambda_{\mathrm{p}} / \xi_{\mathrm{p}}\right)}{R_{\mathrm{c}}} \equiv-\frac{R_{\mathrm{c}}}{S} F_{\mathrm{b}}=-0.35 \mathrm{dyn}_{\mathrm{cm}}^{-1}
$$

using a value of $\frac{R_{c}}{S} \sim \ln 2$ for an assumed spatially uniform distribution of the fluxoids.

The electron currents at the bottom of the crust are however subject to diffusion processes, the rate of which would set a maximum limiting speed $v_{\max }$ for the fluxoids end points to move. 
We, therefore, assume that whenever $v_{\mathrm{p}}<v_{\max }$ the fluxoids remain straight and no curvature force will be acting on them $\left(F_{\mathrm{c}}=0\right)$, since their end points are also able to move with the same speed. In the opposite case, when $v_{\mathrm{p}} \geq v_{\max }$, the fluxoids would be bent outward and the force $F_{\mathrm{c}}$ as in Eq. 7 would be effective. This maximum drift velocity $v_{\max }$ for the magnetic flux in the crust is estimated, based on the Ohmic diffusion alone, as

$$
v_{\max } \sim \frac{R}{\tau}=3.18 \times 10^{-9}\left(\frac{\tau}{10^{7} \mathrm{yr}}\right)^{-1} \mathrm{~cm} \mathrm{~s}^{-1}
$$

where $R=10^{6} \mathrm{~cm}$ is the radius of a neutron star, and $\tau$ is the assumed time scale, in units of yr, for the decay of the magnetic field in the crust. A larger value for $v_{\max }$ may be expected if the Hall drift of the magnetic flux at the bottom of the crust is also taken into account.

On the other hand, the collective rigidity $g$ f the fluxoids lattice due to mutual repulsive forces between them might react to a deformation 16 . The force $F_{\mathrm{c}}$ associated with even a piece of the lattice of a size of an inter-vortex spacing (including some $10^{7}$ flux lines) would be, accordingly, so large that any bending of the lattice is effectively prohibited. The velocity of the fluxoids would then be constrained at all times by the condition $v_{\mathrm{p}} \leq v_{\max }$. We use alternative models to test this possibility as well.

\section{The Models}

The steady-state radial motion of a fluxoid, in the region of interest, is thus determined from the balance equation for all the radial forces acting on it, per unit length, that is 19 .22.

$$
F_{\mathrm{n}}+F_{v}+F_{\mathrm{b}}+F_{\mathrm{c}}=0
$$

which may be rewritten in the form

$$
\alpha \frac{\omega_{-6}}{P_{\mathrm{s}} B_{8}}-\beta v_{\mathrm{p} 7}+\delta=0
$$

where parameters $\alpha, \beta$, and $\delta\left(\equiv F_{\mathrm{b}}+F_{\mathrm{c}}\right)$ would have different values for the alternative models that we have considered, and $v_{\mathrm{p} 7}$ is the fluxoid velocity $v_{\mathrm{p}}$ in units of $10^{-7} \mathrm{~cm} \mathrm{~s}^{-1}$. Recall that $\omega_{-6}$, which is the value of $\omega$ in units of $10^{-6} \mathrm{rad} \mathrm{s}^{-1}$, might have either positive or negative values, as is also the case with $\delta$ in some of the models.

This single equation includes two unknown variables $\omega$ and $v_{\mathrm{p}}$, and represents the azimuthal component of the Magnus equation of motion for the proton vortices. No radial Magnus force acts on the fluxoids, hence the right hand side is set equal to zero, because of the assumed co-rotation of the fluxoids with the proton superconductor. There exist however additional restrictions on the motion of the fluxoids which help to fix the value of one of the variables and solve Eq. 10 for the other. Namely, for a co-moving state $v_{\mathrm{p}}=v_{\mathrm{n}}$ is given and $\omega$ could be determined. In contrast, when $v_{\mathrm{p}}\left(\neq v_{\mathrm{n}}\right)$ is unknown $\omega$ is given as $\omega=\omega_{\mathrm{cr}}$ or $\omega=-\omega_{\mathrm{cr}}$ for $v_{\mathrm{p}}<v_{\mathrm{n}}$ or $v_{\mathrm{p}}>v_{\mathrm{n}}$, respectively. Furthermore, inspection of Eq. 10 indicates that it admits one and only one of the three different solutions, for the given values of $v_{\mathrm{n}}, B_{\mathrm{c}}$, and $P_{\mathrm{s}}$ at any time, namely

$$
\begin{array}{lcc}
\omega=\omega\left(v_{\mathrm{p}}=v_{\mathrm{n}}\right) & \text { iff } & -\omega_{\mathrm{cr}}<\omega<\omega_{\mathrm{cr}} \\
v_{\mathrm{p}}=v_{\mathrm{p}}\left(\omega=\omega_{\mathrm{cr}}\right) & \text { iff } & v_{\mathrm{p}}<v_{\mathrm{n}} \\
v_{\mathrm{p}}=v_{\mathrm{p}}\left(\omega=-\omega_{\mathrm{cr}}\right) & \text { iff } & v_{\mathrm{p}}>v_{\mathrm{n}}
\end{array}
$$

The rate of the flux expulsion out of the core, $\dot{B}_{\mathrm{c}}=-\frac{2}{R_{\mathrm{c}}} B_{\mathrm{c}} v_{\mathrm{p}}$, and the evolution of the stellar surface field $B_{\mathrm{s}}$ (with a decay rate $\dot{B}_{\mathrm{s}}=-\frac{B_{\mathrm{s}}-B_{\mathrm{c}}}{\tau}$ ) are hence uniquely determined from the above force balance equation, given the spin evolution of the star which determines (Eq. 1) the vortex velocity $v_{\mathrm{n}}$ at any time. 


\subsection{The results}

The computed time evolution of $v_{\mathrm{p}}$ and $\omega$ are shown in Fig. 1, together with $v_{\mathrm{n}}$ and $\omega_{\mathrm{cr}}$, as is predicted in one of the models. Characteristically similar results as in Fig. 1 are obtained for the other tested models as well. The fluxoids motion in Fig. 1 is seen to follow three evolutionary phases in which they move slower, together, and faster than the vortices, successively. Transitions
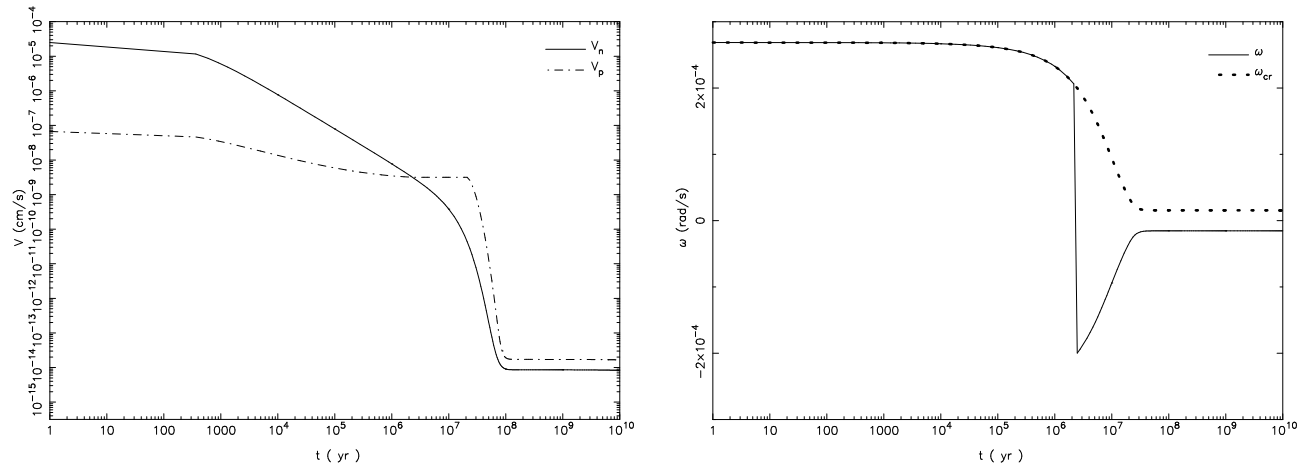

Figure 1: The right panel shows the predicted time evolution of the lag $\omega$ and its critical value $\omega_{\text {cr }}$ in a solitary neutron star according to one of our simulated models. The left panel shows the corresponding evolution of the velocities of the fluxoids $\mathrm{V}_{\mathrm{p}}$, and the vortices $\mathrm{V}_{\mathrm{n}}$. Initial values of $B_{\mathrm{S}}=10^{12.5} \mathrm{G}, B_{\mathrm{c}}=0.9 B_{\mathrm{S}}$, and a value of $\tau=10^{7}$ yr have been used.

between these successive evolutionary phases occur because of the reduction in $v_{\mathrm{n}}\left(\propto \dot{\Omega}_{\mathrm{s}}\right)$ as well as the increase in $P_{\mathrm{s}}$; a final co-moving phase of fluxoids and vortices might also occur for some choices of the initial conditions.

The predicted evolution of the core and surface fields according to the same model is given in Fig. 2. A substantial decrease in the core field occurs at a time $t \gtrsim 10^{7} \mathrm{yr}$, which is expected for the typical average values of $v_{\mathrm{p}} \lesssim 10^{-8} \mathrm{~cm} \mathrm{~s}^{-1}$ during the earlier times, because $\frac{\dot{B}_{\mathrm{c}}}{B_{\mathrm{c}}}=\frac{v_{\mathrm{p}}}{R_{\mathrm{c}}}$ implies that a time period $\Delta t \sim \frac{R_{\mathrm{c}}}{v_{\mathrm{p}}}$ is needed for a major reduction in the core field to occur.
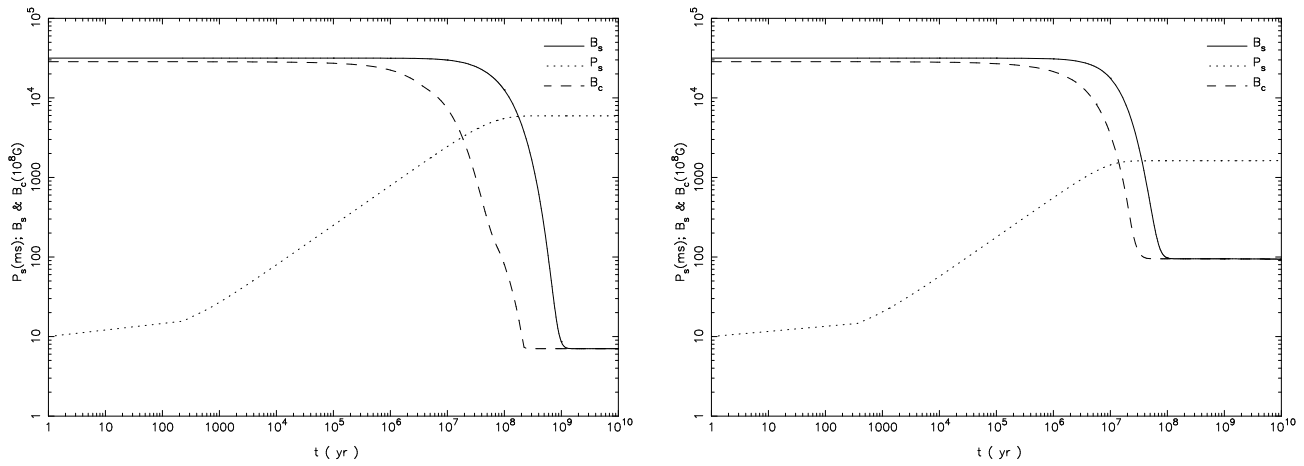

Figure 2: The predicted time evolution of the strength of the magnetic field in the core $B_{\mathrm{c}}$ and at the surface $B_{\mathrm{s}}$, and the spin period $P_{\mathrm{s}}$ in a solitary neutron star according to the same model as in Fig. 1. The right panel (a) is for an assumed value of $\tau=10^{7} \mathrm{yr}$ and corresponds to the results in Fig. 1, while the left panel (b) is for $\tau=10^{8} \mathrm{yr}$.

However, because of the very small magnitude of $v_{\mathrm{p}}$ (although $\gtrsim v_{\mathrm{n}}$ ) and also the reduced value of $B_{\mathrm{c}}$ at later times $B_{\mathrm{c}}$ does not change, substantially, afterwards. The surface field $B_{\mathrm{s}}$ responds to the change in $B_{\mathrm{c}}$ on the assumed decay time scale $\tau$ of the crust. The nontrivial role of the stellar crust in these field evolution models may be seen by comparing Fig. 2a with Fig. 2b, where 
values of $\tau=10^{7}$, and $10^{8}$ yr have been used, respectively. A larger value of $\tau$ tends to maintain the initial $B_{\mathrm{s}}$, hence a larger $\dot{P}_{\mathrm{s}}$ as well as a larger $v_{\mathrm{n}}$, over a more extended period of time. Consequently, smaller final values of $B_{\mathrm{c}}$ and $B_{\mathrm{s}}$ are predicted for the larger assumed values of $\tau$, as is seen in Fig. 2.

Further consideration of the predicted time evolution of the forces on fluxoids, contrasted with the field evolution, reveals that the dominant "driving" force for the flux expulsion is the buoyancy force which is positive throughout the evolution. Accordingly, the overall role of the pinning force in the field decay of neutron stars turns out to be more like a "brake", preventing the flux from being otherwise expelled too rapidly. Our conclusion about the braking role of the pinning force is, however, new and in contradiction with the earlier claims 26 .

\section{References}

1. Baym G., Pethick C., Pines D., Nat., 224, 673 (1969)

2. Bhattacharya D., Datta B., Mon. Not. Royal Astr. Soc., 282, 1059 (1996)

3. de Gennes P. G., "Superconductivity of Metals and Alloys. W. A. Benjamin, Inc., New York. (1966) p. 79

4. Ding K. Y., Cheng K. S., Chau H. F., Astrophys. J., 408, 167 (1993)

5. Flowers E., Ruderman M. A., Astrophys. J., 215, 302 (1977)

6. Ginzburg V. L., Kirzhniz D. A., Nat., 220, 148 (1968)

7. Goldreich P., Reisenegger A., Astrophys. J., 395, 250 (1992)

8. Harvey J. A., Ruderman M. A., Shaham J., Phys. Rev. D 33, 2084 (1986)

9. Hoffberg M., Glassgold A. E., Richardson R. W., Ruderman M., Phys. Rev. Lett., 24, 775 (1970)

10. Jahan-Miri M., Mon. Not. Royal Astr. Soc., 283, 1214 (1996)

11. Jahan-Miri M., PhD thesis, Indian Institute of Science, Bangalore (1996)

12. Jahan-Miri M., to appear in Astrophys. J., (1999)

13. Jahan-Miri M., Bhattacharya D., Mon. Not. Royal Astr. Soc., 269, 455 (1994)

14. Jones P. B., Mon. Not. Royal Astr. Soc., 228, 513 (1987)

15. Jones P. B., Mon. Not. Royal Astr. Soc., 233, 875 (1988)

16. Jones P. B., Mon. Not. Royal Astr. Soc., 253, 279 (1991)

17. Mendell G., Astrophys. J., 380, 515 (1991)

18. Muslimov A. G., Tsygan A. I., Astrophys. Sp. Sci., 115, 43 (1985)

19. Nozières P., Vinen W. F., Phil. Mag., 14, 667 (1966)

20. Phinney E. S., Kulkarni S. R., Ann. Rev. Astron. Astrophys., 32, 591 (1994)

21. Pines D., Alpar M. A., in Pines D., Tamagaki R., Tsuruta S., eds, Proc. US-Japan SENS 90, The Structure and Evolution of Neutron Stars. Addison-Wesley Pub., (1992) p. 7

22. Poole C. P., Farach H. A., Creswick R. J., Superconductivity, Academic Press Inc. (1995)

23. Ruderman M., Ann. Rev. Astron. Astrophys., 10, 427 (1972)

24. Ruderman M., in Fruchter A. S., Tavani M., Backer D. C., eds, A Decade of Surprises. ASP Conference series vol. 72, (1995) p. 276

25. Sauls J. A., in Ögelman H., van den Heuvel E. P. J., eds, Proc. NATO ASI 262, Timing Neutron Stars. Kluwer, Dordrecht, (1989) p. 457

26. Srinivasan G., Bhattacharya D., Muslimov A. G., Tsygan A. I., Curr. Sci., 59, 31 (1990)

27. Taam R. E., van den Heuvel E. P. J., Astrophys. J., 305, 235 (1986)

28. Tilley D. R., Tilley J., Superfluidity and superconductivity, Hilger, Boston (1986) 